\title{
Low prevalence of relevant findings in inappropriate echocardiograms and discordant perceptions between cardiologists and patients
}

\author{
J.R. Lopes ${ }^{1,2}$, A.C. Oliveira ${ }^{1}$, V.G. Rios ${ }^{2}$ and L.C.L. Correia ${ }^{1,3}$ \\ ${ }^{1}$ Escola Bahiana de Medicina e Saúde Pública, Brotas, Salvador, BA, Brasil \\ ${ }^{2}$ Cardioclin, Conceição do Coité, BA, Brasil \\ ${ }^{3}$ Hospital São Rafael, Salvador, BA, Brasil
}

\begin{abstract}
Complementary examinations are "inadequate" whenever the likelihood of benefits from their indication is lower than the negative results. The low benefit is a result of poor performance in detecting relevant changes that lead to improved behavior. However, inadequate examinations are prevalent and little is known about patients' notions of the usefulness of such indications. The aim of this study was to describe relevant findings in inappropriate echocardiograms and to assess the level of agreement between patients and cardiologists regarding their usefulness. Adults without known cardiovascular disease who were referred for echocardiogram by inappropriate criteria according to the American College of Cardiology were selected. Relevant findings were defined by any change in the degree of moderate to severe, according to the American Society of Echocardiography. We tested the level of agreement between the patients who underwent echocardiographic examination and the physicians who requested the exam through a standard questionnaire. Five hundred patients were included, with average age of $52 \pm 17$ years (47\% males). Only 17 patients had any relevant changes $(3.4 \%, 95 \% \mathrm{Cl}=2$ to $5.4 \%)$. The most frequent alterations included valve changes in 8 and diastolic dysfunction grade II in 6 patients. Eighty-seven examinations were performed to determine the level of agreement between patients and cardiologists. For the question "Is this test really necessary?", 92\% of patients responded positively, compared with $5 \%$ of cardiologists (Kappa negative 0.04 ; P=0.01). The frequency of relevant findings was low in inadequate echocardiograms and patients and cardiologists had a different perception regarding its usefulness.
\end{abstract}

Key words: Echocardiogram; Inappropriate; Utility; Patients; Cardiologists

\section{Introduction}

Echocardiography is an imaging method that allows for the diagnosis and management of cardiovascular diseases $(1,2)$, representing approximately half of the diagnostic tests performed in cardiac imaging services (3). In many cases, it is used as a screening tool in patients with low cardiovascular risk (4).

Some studies showed a marked increase in the number of echocardiogram requests in recent years (6-8\% per year) $(3,5)$. In Brazil (2010), 715,655 elective echocardiograms were performed in outpatients in the Unified Health System (SUS), and 425.317 (59\%) of them were performed in the southeastern region of the country, with an estimated cost of over $\mathrm{R} \$ 30$ million (6), contributing to a progressive increase of public health costs (7).

To improve clinical practice, reduce unnecessary exams, and optimize the cost-effectiveness relationship, the American College of Cardiology and the American Society of Echocardiography published the Appropriate Use Criteria for requesting echocardiograms (8). Different clinical scenarios were classified as appropriate, uncertain or inappropriate according to a score system based on the reason for the request. Inappropriate exam requests are those with inadequate indication and that will likely not provide benefit to the patient. Since then, some actions have been taken to try to improve this scenario and reduce the number of inappropriate echocardiograms, and the Choosing Wisely campaign was launched $(9,10)$.

In some articles, the varied prevalence of important findings in inappropriate echocardiograms may reflect the study population (11-14). Barbosa et al. (15) did not find a difference between public and private hospitals in the adequacy of transthoracic echocardiogram requests for outpatients.

Therefore, the objectives of this study were to i) describe the frequency of relevant findings in echocardiograms with

Correspondence: J.R. Lopes: <jrpintolopes@yahoo.com.br> 
inappropriate indication and ii) to evaluate the agreement between physicians and patients regarding their perception of the utility of the echocardiographic tests performed.

\section{Material and Methods}

\section{Sample selection}

From September 2016 until April 2017, all patients who underwent echocardiograms in a private office in the city of Conceição do Coité (Brazil) were screened for the study. Inclusion criteria were defined as follows: age $>18$ years, absence of previously diagnosed heart disease, echocardiogram considered inappropriate, acceptance to participate in the study, and signature of the free and informed consent form.

Prior heart disease was defined based on the known diagnosis of acute or chronic heart disease, characterized by structural change in morphology and/or reduction in functional heart capacity. Inadequate echocardiogram was defined according to the appropriate use criteria of the American Society of Echocardiography (Appropriate Use Criteria for Echocardiography, 2011) (8). The concept of appropriateness establishes that an "appropriate imaging test is one in which the incremental information combined with clinical judgment exceeds the possible negative consequences for a large margin of specific indications, in which the procedure is considered acceptable and reasonable". The request indications are classified as appropriate, uncertain, and inappropriate, according to a score system (9-7, 6-4, and 3-1, respectively) (8). Recently, a new nomenclature was proposed: an appropriate test would be called "test with appropriate care", an uncertain test would be called "test with possible appropriate care", and inappropriate test would be called "test with rarely appropriate care" (9).

\section{Study protocol}

Patients arriving at the office to undergo echocardiography were individually informed about the study and invited to participate. Study details were explained, and patients received the free and informed consent form to read; they were included in the study only after their formal written consent.

Data collection was performed as an interview in an office environment, with the completion of a structured data collection sheet preferably by the patients or with the assistance of the investigator or an accompanying person in case the patient was illiterate. The information was related to patient identification, anamnesis, details of symptoms, and elucidation of the reason for the test request (with the aid of the request guide signed by the attending physician when available). A question was made regarding the expectation of the patient and cardiologist on the utility of the echocardiogram result. Numerical identification codes for the patient and requesting physician were created to protect the names of research participants.
As the service cardiologists were also interviewed, they also received the free and informed consent form.

After the interview, each patient was referred to another office to undergo the test. The physician who performed the echocardiogram did not participate in the study and, therefore, did not have access to the patients' data sheet. Next, two copies of the test were printed, one given to the patient to be sent to the physician, and the other attached to the patient's file. Then, the investigator started the analysis of each exam to classify its adequacy using the list with the 98 indications for the transthoracic echocardiogram, according to the Appropriate Use Criteria for Echocardiography (2011) (8).

\section{Definition of relevant findings}

The echocardiographic findings showing changes compatible with structural or functional heart diseases were defined as relevant in moderate to severe degrees according to the recommendations of the American Society of Echocardiography $(1,16)$. The findings considered relevant in inappropriate echocardiograms are as follows: 1) systolic dysfunction of the left ventricle (Simpson) when the ejection fraction is $<40 \%$; 2) diastolic dysfunction of the left ventricle (grades II-IV); 3) change in the left ventricular contractility (hypokinesia, akinesia, or dyskinesia); 4) valvar changes (at least moderate), mitral stenosis (valvar area $<1.5 \mathrm{~cm}^{2}$ ); mitral insufficiency with vena contracta value $>3 \mathrm{~mm}$ and/or jet/AE area ratio $>20 \%$; aortic stenosis with mean gradient $>30 \mathrm{mmHg}$ or maximum gradient $>50 \mathrm{mmHg}$; aortic insufficiency when the ratio between the regurgitant jet width/VSVE $>25 \%$ or the regurgitant jet length $>2 \mathrm{~cm}$ of the aortic valve is demonstrated; tricuspid insufficiency as expressed in the ratio between jet volume/right atrium $>50 \%$ and/or vena contracta width $>1 \mathrm{~cm} ; 5$ ) dilation of the left ventricle: men (diastolic diameter $>64 \mathrm{~mm}$ and systolic diameter $>44 \mathrm{~mm}$ ) and women (diastolic diameter $>57 \mathrm{~mm}$ and systolic diameter $>39 \mathrm{~mm}$ ); 6) dilation of the right ventricle: (baseline diameter $>41 \mathrm{~mm}$ and/or mean diameter $>35 \mathrm{~mm}$ ); 7) pulmonary arterial hypertension when the systolic pressure of the pulmonary artery is $>45 \mathrm{mmHg}$; 8 ) cardiac masses or tumors: (eg, atrial myxomas, vegetations, and intracavitary thrombi); 9) changes in the pericardium, pericardial fluid involving the whole heart, and signs of pericarditis with thickening of $>2 \mathrm{~mm}$; 10) congenital heart disease: (eg, interatrial communication, ventricular septal defect, aortic stenosis, pulmonary stenosis, and subaortic stenosis).

\section{Perception of cardiologists and patients}

Comparison between proportions of questions asked to physicians and patients was evaluated in 97 tests, since only service cardiologists participated in this phase of the study. Three equal questions were asked to patients and cardiologists. The first question ("In your opinion, this test is") had two possible answers: "little necessary" or 
"very necessary". The second question ("What is the likelihood of this test to detect an important change?") also had two possible answers: "low likelihood" or "high likelihood". The third question ("You are undergoing this test") had three possible answers: "for checkup (no symptoms)", "to investigate the cause of symptoms", or "to evaluate existing disease".

\section{Statistical analysis}

To evaluate the frequency of relevant findings in inappropriate echocardiograms (first objective), the study sample size was calculated by estimating a $5 \%$ prevalence of useful tests to obtain a $95 \% \mathrm{Cl}$ (range: $\pm 2 \%$ ). Thus, 460 inappropriate echocardiograms were required.

Regarding the analysis of agreement between cardiologists and patients on their perceptions of the utility of inappropriate echocardiograms (second objective) and given the final number (97) of tests requested by staff cardiologists, a sample size was calculated a posteriori (Kappa $=0.67$ ) to achieve a statistical power of $80 \%$ (alpha $=5 \%$ ).

Categorical variables were reported as either frequencies or percentages. The chi-squared test was used to compare the inappropriate test requests with sample characteristics. $P$ values $<0.05$ were considered significant for all statistical tests. All statistical analyses were performed using the Statistical Package for the Social Sciences (SPSS, v.17, USA) software.

The tests were performed by a single medical echocardiographist who used an Echocardiography Phillips $\mathrm{HD}^{\circledR}{ }^{\circledR}$ (Bothell, USA) equipment. The study project was approved by the Research Ethics Committee of the Escola Bahiana de Medicina e Saúde Pública (No. 1,661,798; CAAE \# 55513416.8.0000.5544). The study was conducted in accordance with the National Health Council (CNS) resolution (\# 466/2012), meeting the guidelines and norms regulating research involving human beings.

\section{Results}

\section{Sample characteristics}

From September 2016 to April 2017, 1075 echocardiogram tests were performed in this cardiology service; however, four of these patients refused to participate in the study. A total of 1071 patients signed the free and informed consent form; 569 of them had their tests classified as inappropriate (53\%), and 69 of these were excluded because heart disease was previously diagnosed. Thus, the study sample consisted of 500 patients (age: $52 \pm 17$ years), and their distribution between men and women was balanced. No test was covered by SUS; about half $(51 \%)$ of the tests were covered by private health plans and the remainder were paid by the patients. In this group, $11 \%$ were illiterate and only $26 \%$ had university education (Table 1).
Most patients were asymptomatic and 39\% had symptoms, including dyspnea, chest pain, palpitation, edema in the lower limbs, dizziness, and syncope. The percentage of patients with symptoms related to the cardiovascular system was significant. However, their conditions did not imply a significant likelihood of heart disease, not meeting the criteria for appropriate use of the test.

Half of the patients presented some type of morbidity, and systemic arterial hypertension was the most prevalent. However, cases of disabling disease were not observed (39\% of patients had regular physical activity). A total of $44 \%$ of patients had regular use of medications, most of which were antihypertensive drugs (Table 1).

\section{Relevant echocardiographic findings}

Only 17 (3.4\%) patients presented some relevant change on the echocardiogram $(95 \% \mathrm{Cl}=2.0-5.4 \%)$. The most frequent changes were moderate valve changes (8 patients), followed by diastolic dysfunction grade II (6 patients), and moderate (two patients) and marked (two patients) left ventricular systolic dysfunctions. Findings corresponding to pulmonary arterial hypertension, dilation of the right ventricle, cardiac masses or tumors as well as pericardial changes were not observed in the tests (Table 2).

Relevant echocardiographic findings were observed only in three types of indications for inappropriate echocardiogram request. Most of these findings occurred in tests whose indication corresponded to a routine evaluation of systemic arterial hypertension (with no sign or symptom of hypertensive heart disease; 13 tests), followed by routine perioperative evaluation of ventricular function (with no sign or symptom of cardiovascular disease; 3 tests), and indication of initial evaluation of ventricular function (screening; with no sign or symptom of cardiovascular disease; 1 test).

\section{Agreement in perception by cardiologists and patients}

Most patients (92\%) and few cardiologists (5\%) responded that the test was very necessary, with agreement of only $9 \%$ (negative Kappa of 0.04 ; statistically significant for disagreement; $\mathrm{P}=0.01$ ). Most patients $(90 \%)$ and few cardiologists $(6 \%)$ answered that there was a great chance of finding a significant change, with agreement of only $11 \%$ (negative kappa of 0.04 ; statistically significant for disagreement; $P=0.021$ ). Regarding the question about the reason for undergoing the test, $65 \%$ of patients and $42 \%$ of cardiologists answered "checkup (no symptom)"; whereas $31 \%$ of patients and $18 \%$ of cardiologists answered "to investigate the cause of symptoms"; and $4 \%$ of patients and $40 \%$ of cardiologists answered "evaluation of an existing disease" (agreement: 51\%; Kappa=0.24; $\mathrm{P}<0.001$; Table 3). 
Table 1. Baseline characteristics of the study population.

\begin{tabular}{|c|c|}
\hline Clinical characteristics & Frequencies (\%) \\
\hline Sample & 500 \\
\hline Age (years, mean \pm SD) & $52 \pm 17$ \\
\hline Males & $236(47)$ \\
\hline Health plan & $255(51)$ \\
\hline \multicolumn{2}{|l|}{ Patients education } \\
\hline Illiterate & $53(11)$ \\
\hline Primary school & $113(24)$ \\
\hline High school & $184(39)$ \\
\hline University & $126(26)$ \\
\hline Cardiovascular symptoms & $197(39)$ \\
\hline Dizziness & $73(15)$ \\
\hline Palpitations & $67(13)$ \\
\hline Dyspnea & $57(11)$ \\
\hline Chest pain & $37(7)$ \\
\hline Edema in lower limbs & $13(3)$ \\
\hline Syncope & $7(1)$ \\
\hline Presence of chronic disease or clinical change & $256(51)$ \\
\hline Systemic arterial hypertension & $224(45)$ \\
\hline Dyslipidemia & $67(13)$ \\
\hline Preoperative & $47(9)$ \\
\hline Diabetes mellitus & $38(8)$ \\
\hline Heart murmur & $4(1)$ \\
\hline Renal insufficiency & $3(0.6)$ \\
\hline Systemic arterial hypertension & $224(45)$ \\
\hline Dyslipidemia & $67(13)$ \\
\hline Preoperative & $47(9)$ \\
\hline Diabetes mellitus & $38(8)$ \\
\hline Heart murmur & $4(1)$ \\
\hline Renal insufficiency & $3(0.6)$ \\
\hline Physical activity & $195(39)$ \\
\hline Alcoholism & $53(11)$ \\
\hline Smoking & $24(5)$ \\
\hline \multicolumn{2}{|l|}{ Medications used } \\
\hline At least one of the medicines & $219(44)$ \\
\hline Angiotensin receptor blocker & $121(24)$ \\
\hline Diuretic & $69(14)$ \\
\hline Beta blocker & $65(13)$ \\
\hline Calcium channel blocker & $35(7)$ \\
\hline Angiotensin Converting Enzyme Inhibitor & $25(5)$ \\
\hline \multicolumn{2}{|l|}{ Reasons for requesting the echocardiogram } \\
\hline Routine assessment of systemic hypertension without symptoms or signs of HHD & $204(40.8)$ \\
\hline Initial evaluation of ventricular function (screening) & $185(37)$ \\
\hline Peri-operative routine evaluation & $47(9.4)$ \\
\hline Assessment of dizziness or pre-syncope & $31(6.2)$ \\
\hline Assessment of premature atrial contraction & $19(3.8)$ \\
\hline Evaluation of ventricular function with normal pre-evaluation & $8(1.6)$ \\
\hline Evaluation of asymptomatic sinus bradycardia & $3(0.6)$ \\
\hline Routine assessment of vestigial valve regurgitation & $2(0.4)$ \\
\hline Initial assessment with no sign or symptom of structural heart disease & $1(0.2)$ \\
\hline
\end{tabular}

HHD: hypertensive heart disease. 
Table 2. Proportion of relevant findings in inappropriate echocardiograms.

\begin{tabular}{llr}
\hline Relevant findings & Frequencies $(\%)$ & $95 \% \mathrm{Cl}$ \\
\hline Tests with at least one relevant finding & $17(3.4)$ & $2.0-5.4$ \\
Systolic dysfunction & $2(0.4)$ & $0.05-1.4$ \\
$\quad$ Moderate & $2(0.4)$ & $0.05-1.4$ \\
Important & & \\
Diastolic dysfunction & $6(1.2)$ & $0.44-2.6$ \\
$\quad$ Grade II & 0 & \\
Grade III & 0 & $0.01-1.1$ \\
$\quad$ Grade IV & & $0.05-1.4$ \\
Change in contractility & $1(0.2)$ & $0.7-3.1$ \\
$\quad$ Hypokinesia & $2(0.4)$ & $0.22-2.6$ \\
Akinesia & 0 & $0.01-1.1$ \\
Dyskinesia & $8(1.6)$ & $0.01-1.1$ \\
Valvar change & $4(0.8)$ & $0.01-1.1$ \\
Moderate mitral insufficiency & $1(0.2)$ & $0.01-1.1$ \\
Important mitral insufficiency & $1(0.2)$ & \\
Moderate aortic stenosis & $1(0.2)$ & \\
Moderate aortic insufficiency & $1(0.2)$ & \\
Important aortic insufficiency & & $0.01-1.1$ \\
Dilatation of the left ventricle & 0 & \\
Moderate & $2(0.4)$ & \\
Important & 0 & \\
Dilatation of the right ventricle & 0 & \\
Moderate & 0 & \\
Important & & \\
Pulmonary arterial hypertension & $0.2)$ & \\
Congenial heart disease & & \\
$\quad$ Subvalvar aortic stenosis & & \\
Cardiac masses or tumors & & \\
\hline
\end{tabular}

Table 3. Agreement between physicians' and patients' perceptions regarding the utility and reason of tests.

\begin{tabular}{lcccc}
\hline Questions & Patients (\%) & Physicians (\%) & Kappa values & P values \\
\hline 1. Test was much needed & 92 & 5 & -0.04 & 0.01 \\
2. High chance of identifying a significant cardiac change & 90 & 6 & -0.04 & 0.01 \\
3. Reason for the test & & & & \\
$\quad$ Checkup (no symptoms) & 65 & 42 & 0.24 & $<0.001$ \\
$\quad$ Investigation of the cause of symptoms & 31 & 18 & \\
$\quad$ Evaluation of an existing disease & 4 & 40 & & \\
\hline
\end{tabular}

\section{Discussion}

In the present study, two observations were evident: first, the frequency of relevant findings obtained in echocardiograms requested in inappropriate situations was very low, reinforcing the little utility of these tests; second, there is no agreement between the opinions of cardiologists and their patients regarding utility of these tests, suggesting that the physicians' notion of test utility is not adequately communicated to their patients.
The characteristics of the study population and the situations in which the tests were requested justify the above result. These tests were performed on an elective basis in patients free from previously diagnosed heart disease. In addition, a significant number of patients had initial ventricular function evaluation (screening) as indication, with no sign or symptom of cardiovascular disease. This information shows that echocardiographic tests, which are classified as inappropriate, contribute little to discover important cardiac changes. The impact of these 
results is likely to be small in relation to future clinical decisions that imply changes in medical conduct regarding patient care. The reason is that the rate of change in the clinical management of patients is considered low even after tests classified as appropriate (17). Therefore, our data reinforce their classification as inappropriate as proposed by the American College of Cardiology.

Given the potential for unintended consequences, efforts should be made to reduce the number of inappropriate echocardiograms such educational interventions $(18,19)$, consulting the appropriateness criteria, use of authorized protocols, and auditing laboratories with evaluation of the echocardiographic requests (20). Recently, Bhatia et al. of the Echo WISELY Trial published an investigator-blind randomized clinical trial, showing the efficacy of educational intervention to reduce inappropriate echocardiograms in outpatients (21). The ultimate goal of these educational interventions has been to benefit patients, as negative consequences are prevented, and reduce health costs.

The proportions of echocardiographic findings considered important obtained in our study was lower than that found in the study published by Koshy et al. (11); these authors found that the prevalence of findings considered abnormal $(51 \%)$ in tests classified as appropriate was higher than in those classified as inappropriate (38\%) $(P=0.013)$. A study by Ward et al. (12) evaluated echocardiograms performed at the outpatient clinic of a university hospital, where new and important abnormalities in appropriate tests $(40 \%)$ were more common than in inappropriate tests $(17 \%)(P<0.001)$. Kirkpatrick et al. $(13)$ reported a $20 \%$ rate for clinically important new or unexpected echocardiographic findings in tests classified as inappropriate. Similar results were published by Ward et al. (14), who observed a high frequency of new and important abnormalities on echocardiogram tests (classified as inappropriate) carried out in academic centers (16\%) and community care centers $(15 \%)$. In the Troms $\varnothing$ study, in which patients were submitted to echocardiography as screening, a low prevalence of abnormal cardiac findings $(7.6 \%)$ was observed, and valve disease was the most common finding (22).

However, it is important to emphasize that patients with known diagnosis of heart disease were included in all the studies cited above, differently from our study. The clinical conditions of these patients were also different because patients who were hospitalized or in situations of

\section{References}

1. Cheitlin MD, Alpert JS, Armstrong WF, Aurigemma GP, Beller GA, Bierman FZ, et al. ACC/AHA guidelines for the clinical application of echocardiography: executive summary. A report of the American College of Cardiology/American Heart Association Task Force on practice guidelines (Committee on Clinical Application of Echocardiography). acute or decompensated illnesses were also included in the other studies. This contributed to the difference in the proportion of echocardiographic findings in relation to our study, which is the first Brazilian evaluation of the frequency of findings considered relevant; in this study, patients without the diagnosis of heart disease submitted to elective echocardiograms classified as inappropriate were included.

Some issues should be mentioned on the disagreement between the views of cardiologists and their patients regarding the clinical utility of the tests and reason to request them. Given the current scenario of medical practice, in which little dialogue occurs between doctors and patients, patients are not sufficiently informed about the consequences of undergoing medical tests. In the study published by Barros et al. (23) on empathy in the doctor-patient relationship in the context of public and private health, low agreement was found in the perception of medical empathy in the public (22\%) and private $(34.3 \%)$ sectors. This suggests that a fragile relationship with little dialogue is established during medical care. Initiatives such as the Choosing Wisely strategy (10) are an advancement, as they try to counteract this scenario by broadening the understanding on inherent aspects of earning interest in the medical practice, allowing the patient to act in decisions related to their health. In addition, analyses have identified situations or factors that may contribute to the excessive use of tests in cardiology services, as cultural reasons also influence these requests. These factors can be classified into clinical or financial, and the reasons may be legal or juridical (24). This is also the first Brazilian study evaluating the agreement between cardiologists and patients regarding inappropriate echocardiograms.

The main limitation of this study is its low representativeness, as it was obtained from an outpatient clinic (convenient sample). Therefore, the applicability of our findings is restricted to populations with characteristics similar to those of our sample. Regarding the issue of doctor-patient communication, our findings may raise the possibility that patients are unaware of the utility of inappropriate tests.

In conclusion, the present study showed that elective echocardiograms requested in inappropriate situations and performed in patients without a diagnosis of heart disease present a low frequency of relevant findings. In addition, patients and cardiologists may have very different perceptions regarding utility of inappropriate tests.
Developed in collaboration with the American Society of Echocardiography. J Am Coll Cardiol 1997; 29: 862-879, doi: 10.1016/S0735-1097(96)90000-5.

2. Garbi M, McDonagh $T$, Cosyns B, Bucciarelli-Ducci $C$, Edvardsen T, Kitsiou A, et al. Appropriateness criteria for cardiovascular imaging use in heart failure: report of 
literature review. Eur Heart J Cardiovasc Imaging 2015; 16 : 147-153, doi: 10.1093/ehjci/jeu299.

3. Pearlman AS, Ryan T, Picard MH, Douglas PS. Evolving trends in the use of echocardiography: a study of medicare beneficiaries. J Am Coll Cardiol 2007; 49: 2283-2291, doi: 10.1016/j.jacc.2007.02.048.

4. Colla $\mathrm{CH}$, Sequist TD, Rosenthal MB, Schpero WL, Gottlieb DJ, Morden NE. Use of non-indicated cardiac testing in lowrisk patients: Choosing Wisely. BMJ Qual Saf. 2015; 24(2): 149-53, doi: 10.1136/bmjqs-2014-003087.

5. Blecker S, Bhatia RS, You JJ, Lee DS, Alter DA, Wang JT, et al. Temporal trends in the utilization of echocardiography in Ontario, 2001 to 2009. JACC Cardiovasc Imaging 2013; 6: 515-522, doi: 10.1016/j.jcmg.2012.10.026.

6. Barachi LB, Barbosa FC, Moscavitch SD, Pilon BC, Pissinati $\mathrm{GV}$, Mesquita $\mathrm{ET}$, et al. Inadequate request of transthoracic echocardiography according to the guidelines of the Brazilian Society of Cardiology. Arq Bras Cardiol 2012; 99: 952-954, doi: 10.1590/S0066-782X2012001300012.

7. Douglas PS. Quality in echocardiography: choosing to succeed. J Am Soc Echocardiogr 2008; 21: 1016-1017, doi: 10.1016/j.echo.2008.07.010.

8. ACCF/ASE/AHA/ASNC/HFSA/HRS/SCAI/SCCM/SCCT/ SCMR. Appropriate Use Criteria for Echocardiography. A Report of the American College of Cardiology Foundation Appropriate Use Criteria Task Force, American Society of Echocardiography, American Heart Association, American Society of Nuclear Cardiology, Heart Failure Society of America, Heart Rhythm Society, Society for Cardiovascular Angiography and Interventions, Society of Critical Care Medicine, Society of Cardiovascular Computed Tomography, and Society for Cardiovascular Magnetic Resonance Endorsed by the American College of Chest Physicians. J Am Coll Cardiol 2011; 57: 1126-1166, doi: 10.1016/j.jacc.2010.11.002.

9. Bhatia RS, Alabousi M, Dudzinski DM, Weiner RB. Appropriate use criteria: a review of need, development and applications. Expert Rev Cardiovasc Ther 2016; 14: 281-290, doi: 10.1586/14779072.2016.1131125.

10. Bhatia RS, Ivers N, Yin CX, Myers D, Nesbitt G, Edwards J, et al. Design and methods of the Echo Wisely (Will Inappropriate Scenarios for Echocardiography Lessen Significantly) study: An investigator-blinded randomized controlled trial of education and feedback intervention to reduce inappropriate echocardiograms. Am Heart J 2015; 170: 202-209, doi: 10.1016/j.ahj.2015.04.022

11. Koshy TP, Rohatgi A, Das SR, Price AL, deluna A, Reimold $\mathrm{N}$, et al. The association of abnormal findings on transthoracic echocardiography with 2011 appropriate use criteria and clinical impact. Int J Cardiovasc Imaging 2015; 31: 521-528, doi: 10.1007/s10554-014-0582-x.

12. Ward RP, Mansour IN, Lemieux N, Gera N, Mehta R, Lang RM. Prospective evaluation of the clinical application of the American College of Cardiology Foundation/American Society of Echocardiography Appropriateness Criteria for transthoracic echocardiography. JACC Cardiovasc Imaging 2008; 1: 663-671, doi: 10.1016/j.jcmg.2008.07.004
13. Kirkpatrick JN, Ky B, Rahmouni HW, Chirinos JA, Farmer $\mathrm{SA}$, Fields AV, et al. Application of appropriateness criteria in outpatient transthoracic echocardiography. J Am Soc Echocardiogr 2009; 22: 53-59, doi: 10.1016/j.echo.2008.10.020.

14. Ward RP, Krauss D, Mansour IN, Lemieux N, Gera N, Lang RM, et al. Comparison of the clinical application of the American College of Cardiology Foundation/American Society of Echocardiography Appropriateness Criteria for outpatient transthoracic echocardiography in academic and community practice settings. J Am Soc Echocardiogr 2009; 22: 1375-1381, doi: 10.1016/j.echo.2009.08.005.

15. Barbosa FC, Mesquita ET, Barachi LB, Salgado A, Kazuo R, Rosa ML, et al. Comparison of echocardiography request appropriateness between public and private hospitals. Arq Bras Cardiol 2011; 97: 281-288, doi: 10.1590/S0066782X2011005000106.

16. Lang RM, Badano LP, Mor-Avi V, Afilalo J, Armstrong A, Ernande $\mathrm{L}$, et al. Recommendations for cardiac chamber quantification by echocardiography in adults: an update from the American Society of Echocardiography and the European Association of Cardiovascular Imaging. J Am Soc Echocardiogr 2015; 28: 1-39.e14, doi: 10.1016/j.echo.2014. 10.003.

17. Matulevicius SA, Rohatgi A, Das SR, Price AL, DeLuna A, Reimold SC. Appropriate use and clinical impact of transthoracic echocardiography. JAMA Intern Med 2013; 173: 1600-1607, doi: 10.1001/jamainternmed.2013.8972.

18. Bhatia RS, Milford CE, Picard MH, Weiner RB. An educational intervention reduces the rate of inappropriate echocardiograms on an inpatient medical service. JACC Cardiovasc Imaging 2013; 6: 545-555, doi: 10.1016/j.jcmg.2013.01.010.

19. Bhatia RS, Dudzinski DM, Malhotra R, Milford CE, Yoerger Sanborn DM, Picard MH, et al. Educational intervention to reduce outpatient inappropriate echocardiograms: a randomized control trial. JACC Cardiovasc Imaging 2014; 7: 857-866, doi: 10.1016/j.jcmg.2014.04.014.

20. Fonseca R, Marwick TH. How I do it: judging appropriateness for TTE and TEE. Cardiovasc Ultrasound 2014; 12: 22, doi: 10.1186/1476-7120-12-22.

21. Bhatia RS, Ivers NM, Yin XC, Myers D, Nesbitt GC, Edwards $\mathrm{J}$, et al. Improving the appropriate use of transthoracic echocardiography: The Echo WISELY Trial. J Am Coll Cardiol 2017; 70: 1135-1144, doi: 10.1016/j.jacc.2017.06.065.

22. Lindekleiv $H$, Lochen ML, Mathiesen EB, Njolstad I, Wilsgaard T, Schirmer $\mathrm{H}$. Echocardiographic screening of the general population and long-term survival: a randomized clinical study. JAMA Intern Med 2013; 173: 1592-1598, doi: 10.1001/jamainternmed.2013.8412.

23. Barros PS, Falcone EM, Pinho VD. Avaliação da empatia médica na percepção de médicos e pacientes em contextos público e privado de saúde. Arq Ciênc Saúde 2011; 18: $36-43$.

24. Huang $X$, Rosenthal MB. Overuse of cardiovascular services: evidence, causes, and opportunities for reform. Circulation 2015; 132: 205-214, doi: 10.1161/CIRCULATIONAHA.114. 012668. 